\title{
LE “AUGAGNEURISME” OU L'EXPÉRIENCE DU SOCIALISME MUNICIPAL À LYON (1900-1905)
}

L'élection comme maire, après les élections municipales de 1900 remportées par le Bloc républicain, du chirurgien et professeur à la faculté de médecine Victor Augagneur" ouvre une période portant le nom de "socialiste" dans l'histoire municipale lyonnaise. Si cette étiquette politique mérite d'être soulignée à Lyon, c'est que, depuis 1793, les élites économiques et politiques lyonnaises, libérales et peu républicaines jusqu'en 1870 puis républicaines et modérées après cette date, avaient réussi à éviter toute rencontre entre socialisme et pouvoir municipal. Cette volonté des élites lyonnaises d'écarter de l'hôtel-de-ville le socialisme s'explique par le fait qu'elles ont une mémoire politique marquée, depuis la Révolution, par le traumatisme de la municipalité exagérée des "Chalier" qui a gouverné Lyon entre le 8 mars et le 29 mai 1793 et par les conséquences qui en ont découlé2. Si en 1900 l'endiguement des Rouges échoue, c'est que le rapport des forces politiques a brutalement changé à Lyon en cette fin de siècle.

L'affaire Dreyfus, particulièrement le J'accuse de Zola, a rapproché Augagneur, candidat longtemps malheureux à la mairie de Lyon, des socialistes, en particulier ceux du Parti ouvrier français. Devenu dreyfusard, Augagneur reçoit également le soutien de la Ligue des Droits de l'Homme et de la Ligue de Défense républicaine, ligues qui vont être les fers de lance du succès du Bloc républicain aux élections municipales en 1900. L'affaire Dreyfus, en réveillant les tensions cléricales/anticléricales et en rejetant les libéraux à Droite, rompt le consensus centriste et républicain modéré existant à Lyon et jusque-là favorable au maire Gailleton, en fonction depuis les lois municipales de 1881-1884, pour

\footnotetext{
' Né à Lyon en 1855 et mort au Vésinet en 1931, Victor Augagneur, avant de devenir maire de Lyon, fut conseiller municipal en 1888, adjoint en 1890 , démissionnaire en janvier 1892 , candidat malheureux à la mairie la même année et non élu en 1896. Si, jusqu'en 1900, il n'avait jamais affiché de sympathie socialiste, il est le fondateur de la fédération socialiste du Rhône. Député et réélu en 1904, il quitte la mairie de Lyon en 1905 pour être gouverneur de Madagascar jusqu'en 1910. S'il n'arrive pas à récupérer la mairie à son retour, il redevient député du Rhône et est plusieurs fois ministre, pour être après guerre gouverneur de l'AEF.

${ }^{2}$ Pour mieux comprendre la situation politique lyonnaise, consulter mon article Histoire, mémoire et identité politique: l'exemple de la Révolution à Lyon, dans: Annales historiques de la Révolution française 305 (1996) p. 491-509 et ma note de synthèse pour le diplôme d'habilitation à diriger des recherches, Entre violences collectives et mémoire des élites, l'identité d'une grande ville de province, Lyon 1786-1905, Université Lumière Lyon 2, 1997; Bruno BENOIT, L'identité politique de Lyon. Entre violences collectives et mémoire des élites (1786-1905), Paris 1999.
} 
instaurer un clivage Droite-Gauche. Cet affrontement s'avère préjudiciable au maire sortant, car ne s'étant pas impliqué dans le camp dreyfusard, il ne peut compter sur les voix de Gauche, ce qui laisse le champ libre à Augagneur. Quant aux voix de Droite, elles lui font traditionnellement défaut, car elles lui reprochent son ancrage laïque, jacobin et maçonnique.

Devenu maire de Lyon sous l'étiquette socialiste, Victor Augagneur veut rompre avec la majorité sortante qu'il qualifie de "pseudo-républicaine" ${ }^{3}$. Il met alors en place une politique municipale que l'on peut qualifier de socialisme municipal. Cette politique, s'inscrivant dans le cadre et la durée de son mandat municipal qui dure cinq ans, puisqu'il est réélu en 1904 et qu'il l'abandonne en 1905, peut être désignée par le terme d'augagneurisme. Lors de son premier mandat, Augagneur supprime l'octroi frappant "la classe des ouvriers de l'industrie", le remplace par des taxes "sur la classe des propriétaires-rentiers"4 et par la création de régies municipales. Cette politique s'accompagne d'un fort anticléricalisme allant jusqu'à voter en faveur de la fermeture de la basilique de Fourvière et à envoyer une délégation au Congrès universel de la Libre pensée à Rome en 1904.

L'année de sa réélection à la mairie, il est élu député et siège alors aux côtés de Guesde et de Jaurès à l'Assemblée. Cependant en 1905, il conserve son étiquette de socialiste indépendant en n'adhérant pas à la SFIO en avril 1905, ce qui l'expose aux critiques des socialistes locaux. A-t-il compris qu'à Lyon, le pouvoir municipal pour durer a besoin d'éviter de se situer aux extrêmes du paysage politique? Pourtant, il tente, durant son deuxième mandat municipal, de créer un "Grand Lyon", projet qu'il a du mal à faire passer, puisqu'il aurait dû se faire aux dépens des communes socialistes de l'Est lyonnais. Cet agrandissement de Lyon est-il le moyen de trouver une assise électorale qui commence à se dérober à Lyon du fait des réformes entreprises et de son autoritarisme de "proconsul" dénoncé presque dès son arrivée à la mairie ${ }^{5}$ ? Son projet de "Grand Lyon" suscite aussi de vives réactions de la part des communes concernées ${ }^{6}$, ce qui détériore le climat avec les communes voisines et amène Augagneur à se rapprocher des républicains modérés lyonnais qui ont pourtant peu de sympathie pour son étiquette socialiste contraire au consensus lyonnais. Confronté à une situation qui risque de devenir

\footnotetext{
${ }^{3}$ Le Progrès, 23 avril 1900.

${ }^{4}$ Ces expressions sont contenues dans un rapport présenté par Augagneur après son élection, Victor AUGAGNEUR, Suppression de l'octroi et taxes de remplacement, Lyon 1900, p. 4.

${ }_{5}^{5}$ Dans Le Salut public, journal conservateur donc peu favorable à Augagneur, du 26 décembre 1900, le maire est comparé au préfet (la pire injure à Lyon!) Ducros, de sinistre mémoire à Lyon puisqu'il a été le préfet de l'ordre moral: "Comme l'ex-préfet, Monsieur Augagneur est autoritaire, violent et tracassier; comme lui, il ne cesse d'élaborer des mesures vexatoires à l'égard d'une bonne partie de ses concitoyens; comme lui, enfin, il mécontente même ses propres amis par ses manières cassantes et tyranniques". L'article se termine en espérant un départ rapide d'Augagneur.

${ }^{6}$ Bruno BENOIT, Quand Lyon voulut annexer Villeurbanne au début du $\mathrm{XX}^{\mathrm{e}}$ siècle, dans: Bulletin municipal officiel de la ville de Lyon, 5109 et 5167 (mars 1996 et mai 1997).
} 
difficile, mais aussi habité par une dévorante ambition qui lui fait préférer l'opportunité au militantisme, il démissionne de sa fonction de maire en octobre 1905 pour accepter le poste de gouverneur de Madagascar qui lui est proposé.

Mon exposé s'articulera autour de trois temps: d'abord il cherchera à définir ce qu'est l'augagneurisme et en quoi mérite-t-il le nom de socialisme municipal, puis quelles réalisations justifiant ce label ont eu lieu durant les cinq ans du mandat d'Augagneur et enfin quelles lectures peut-on faire de ce socialisme municipal.

\section{Qu'est-ce que l'augagneurisme?}

Le 22 mai 1900, deux jours après avoir été élu maire par 31 voix contre 22 à Antoine Gailleton ${ }^{7}$, ce vote prouvant que l'ancien maire garde des supporters et que les élus de Droite ont voté contre Augagneur le socialiste, ce dernier prononce un discours ${ }^{8}$ dans lequel, après avoir rendu hommage aux forces radicales et socialistes qui l'ont élu, il annonce son programme d'action. Il le place sous les auspices de la libération de l'intelligence et de la conscience humaine, de l'instruction laïque répandue à profusion, de la liberté de pensée, de l'amélioration des conditions économiques pour les classes déshéritées de la fortune. Son programme mérite d'être analysé précisément, car il nous fournit un bon index des principales entrées de l'augagneurisme, expression du socialisme municipal lyonnais.

Son programme réclame, selon sa propre déclaration, la confiance et le concours des élus de sa majorité, la fidélité de la part du personnel municipal, la courtoisie et la loyauté de la part de l'opposition et surtout l'adhésion des Lyonnais. Pour qu'un jour ces idéaux deviennent réalité, il faut que la politique municipale agisse dans les directions suivantes:

1. L'école doit être dégagée de toute influence confessionnelle et doit être émancipatrice. Cette école doit aider dans l'éducation des enfants les parents qui travaillent. De ce fait, Augagneur parle de développer les cantines, les gardiennages le jeudi et lors des vacances, les études et les œuvres postscolaires laïques. Mais l'école doit aussi apporter des connaissances dans le domaine scientifique, dans celui des rapports sociaux, d'où l'idée de développer un enseignement supérieur populaire dans le cadre municipal et un enseignement professionnel. Enfin, l'école doit apprendre, grâce à des manifestations artistiques, "la contemplation de la beauté".

\footnotetext{
${ }^{7}$ Il y a 54 conseillers municipaux, la voix qui manque est un bulletin blanc.

${ }^{8}$ Archives municipales de Lyon (A. M. L.), Bulletin municipal officiel de Lyon, séance du 22 mai 1900.
} 
2. Le travail doit permettre de vivre plus décemment. La commune, en tant qu'employeur, doit servir de modèle au patronat privé en abaissant, pour son personnel, le temps de travail et en augmentant les salaires.

3. L'octroi, parce qu'il est créateur d'une fiscalité inique qui crée des inégalités dans l'alimentation du peuple, doit être supprimé.

4. L'assistance publique doit être développée, car elle est une marque de solidarité sociale. Pour cela, il faut construire des maisons de retraite pour les vieillards et les invalides.

5. Les régies municipales doivent permettre d'exploiter directement les services publics pour le plus grand bien de tous. Sont concernés le service des eaux et le tout à l'égout, mais aussi dans un deuxième temps les théâtres, l'éclairage public, les transports en commun.

6. L'hygiène doit être assurée dans l'intérêt de tous. Les domaines concernés sont l'école, le logement, l'enlèvement et l'incinération des immondices, la construction de maisons mortuaires.

7. Les finances publiques doivent être gérées sainement. De ce fait, finis les grands emprunts. Le recours aux régies municipales doit permettre à la municipalité de compter sur ses propres ressources. De plus, la politique des grands travaux n'a plus lieu d'être, car ce qui est recherché est le soulagement des misères, un plus grand bien-être matériel et moral pour la collectivité communale, mais aussi plus de justice et plus de solidarité pour la "classe la moins fortunée".

Cette politique a été bien accueillie par les Lyonnais, car elle implique une vie meilleure pour la majorité de la population. De ce fait, en mai 1904, à l'occasion des nouvelles élections municipales, il est élu dès le premier tour ${ }^{9}$ ainsi qu'une bonne partie des candidats du Bloc républicain et socialiste qui le soutient. La presse parle d'un triomphe électoral qui acclame les réformes accomplies ${ }^{10}$, renforce l'autorité d'Augagneur sur le Conseil et constitue un véritable plébiscite pour que la municipalité conduite par Augagneur continue à appliquer les grandes réformes de son discours-programme de 1900. L'augagneurisme l'a emporté, puisque lors de l'élection du maire le 15 mai, il recueille 48 voix sur $54^{11}$. En 1904, Augagneur a vidé les rangs de l'opposition de Droite dont les "listes progressistes et réactionnaires" sont en pleine déroute avec seulement 3 élus; il a su aussi regrouper autour de son nom les républicains-socialistes qui n'ont désormais plus d'autre candidat de rechange. Quant aux socialistes dissidents, ils n'arrivent pas à mordre sur l'électorat lyonnais, moins de 200 voix quand ils se présentent, ce qui tendrait à prouver qu'il n'y a pas eu de virage socialiste en 1900, mais

${ }^{9}$ En effet, en 1900, il avait été élu au deuxième tour dans le $6^{\mathrm{e}}$ arrondissement avec 5151 voix, alors qu'en 1904, il en recueille, dès le premier tour, 7246.

${ }^{10}$ Cette expression est due au journal Le Progrès du 2 mai 1904, lendemain du $1^{\text {er }}$ tour.

${ }^{11}$ Il y a une voix à Lavigne, élu sur sa liste dans le $5^{\mathrm{e}}$ arrondissement et 5 bulletins blancs qui comprennent les 3 bulletins de l'opposition libérale, mais aussi 2 bulletins radicaux-socialistes. Faut-il compter parmi eux, celui de l'ancien maire Gailleton, élu du $2^{\mathrm{e}}$ arrondissement, qui a du mal à voter pour son ancien rival? 
plutôt une convergence de facteurs favorables pour Augagneur. Au moment où l'augagneurisme triomphe, est élu, dans le $1^{\text {er }}$ arrondissement, Édouard Herriot qui devient aussi $5^{\mathrm{e}}$ adjoint. $C^{\prime}$ 'est en faveur de ce jeune adjoint, qui ne paraît pas en mesure de lui faire de l'ombre, qu'Augagneur démissionne en octobre $1905^{12}$.

Si son discours ${ }^{13}$ de 1904 insiste sur l'œuvre réalisée et la confiance que les Lyonnais lui ont accordée lors des deux tours de scrutin, il n'annonce, à la différence de celui de 1900, aucun programme, la référence restant le discoursprogramme de 1900 puisqu'il n'a pas encore été totalement réalisé. Il affirme donc en 1904 vouloir poursuivre dans la voie ouverte en 1900 en déclarant que le succès ne le poussera pas à la contemplation satisfaite du passé, mais à agir en regardant l'avenir, afin de se rapprocher de l'idéal radical et socialiste et ce, dans l'intérêt de tous!

Au travers du discours-programme de 1900, réactivé par la déclaration de 1904, il est possible de proposer une première lecture de l'augagneurisme. C'est une politique municipale qui, mettant l'accent prioritairement sur l'aspect social, est bien d'inspiration socialiste quant au vocabulaire utilisé. Victor Augagneur n'hésite pas à nommer précisément ceux à qui sa politique est destinée: le peuple, les ouvriers et les travailleurs d'usine, catégories qu'il oppose aux patrons, propriétaires et adversaires de Droite. Dans son discours du 22 mai 1900 et lors de sa campagne en 1904, il lit les rapports sociaux en conflits de classe et déclare que seule l'action politique est capable de rendre les hommes plus heureux et la société plus juste et plus égalitaire. Ce programme "possibiliste" n'est en aucune façon révolutionnaire, puisque ces changements doivent être obtenus sans violence et en respectant les droits individuels de chaque Lyonnais ${ }^{14}$. Son socialisme est dit municipal parce qu'il pense que les conseils municipaux de Gauche sont en mesure d'administrer les villes aussi correctement que les actionnaires gèrent une société anonyme et que dans leur cas, l'argent n'est pas le mobile à leur activité, mais bien l'intérêt collectif, le dévouement individuel ${ }^{15}$.

\footnotetext{
${ }^{12}$ Il pensait alors pouvoir récupérer son poste à son retour de Madagascar, ce qui fut impossible. Augagneur avait bien mal apprécié le jeune Herriot, puisque celui-ci va rester 52 ans maire de Lyon!

${ }^{13}$ A. M. L., Bulletin municipal officiel de Lyon, séance du 15 mai 1904.

${ }^{14}$ Ibid, séance du 22 mai 1900.

${ }^{15}$ Victor AUGAGNEUR, La régie directe des services publics par les municipalités, dans: Paul PIC, Justin GODART (dir.), Le mouvement économique et social dans la région lyonnaise, 2, Lyon 1905 , p. 83.
} 


\section{L'œuvre accomplie par Augagneur ou le socialisme municipal lyonnais}

Pour apprécier l'œuvre d'Augagneur, laissons lui la parole. En effet, à plusieurs occasions, il va s'exprimer pour faire le bilan de ce qu'il a réalisé. Tel est le cas, lors d'une conférence qu'il donne le 5 juin 1903 dans le cadre de l'Office social de Lyon ${ }^{16}$ qui se veut d'abord un lieu où l'on peut débattre des "questions économiques et sociales: législation du travail, législation commerciale et fiscale, hygiène publique..." et où l'on peut "se documenter sur les réformes sociales accomplies au dehors, les nations voisines nous servant ainsi de champs d'expérience"", mais il veut également être une "école mutuelle de conférenciers populaires" pour éduquer socialement le peuple ${ }^{18}$. Il n'est donc pas étonnant qu'Augagneur vienne donner une conférence dans une telle association qui ne peut que soutenir son action et devant un parterre acquis à ses réformes. Il appelle en 1904 sur sa liste son directeur, Justin Godart ${ }^{19}$, un avocat radical-socialiste, qui est même élu adjoint. La campagne électorale municipale de 1904 donne lieu également à des discours, meetings, prises de parole permettant de saisir quelles ont été les principales réalisations de la première municipalité Augagneur. Tel est le cas lors d'un vin d'honneur à la brasserie Fritz organisé par la Fédération des Comités républicains démocratiques du $2^{\mathrm{e}}$ arrondissement le 11 avril $1904^{20}$.

La grande cewvre de son premier mandat est la suppression totale des octrois, barrières qui, depuis la Révolution, ont souvent poussé la population lyonnaise à se révolter. D'après Augagneur, cette ouvre est tout à la gloire de la ville de Lyon qui a été la seule, depuis 100 ans, à se lancer dans une telle réforme, car à la différence de la loi de 1897 qui n'instaurait qu'une suppression partielle, la

${ }^{16}$ Si l'Office social de Lyon préexiste à la réunion constitutive du 3 février 1903, celle-ci lui donne une autre dimension et de nouveaux statuts, puisqu'il est doté de 7 sections et d'un véritable programme d'action.

${ }^{17}$ A ce propos, un ouvrage donné par Herriot aux Archives municipales de Lyon porte le titre de Socialisme municipal en Angleterre. Cette traduction résumée des articles parus dans le journal "The Times" sous le titre "Municipal socialism" en septembre 1902, Bruxelles 1903, 64 p., comportait sur la page de garde l'inscription manuscrire suivante: "à remettre à M. Herriot. Au cas où cette brochure vous intéresserait, je prie ma tante de bien vouloir vous la remettre". Cette dédicace prouve que le socialisme municipal est dans l'air du temps, que les expériences allemande et anglaise sont très étudiées et que le jeune Herriot est lui aussi intéressé par cette nouvelle approche de la gestion municipale.

${ }^{18}$ Termes du professeur Pic, professeur de législation industrielle à la faculté de droit de Lyon, lors de son allocution à l'assemblée générale constitutive de l'Office social le 3 février 1903, dans: PIC, GODART (dir.), Le mouvement économique 1905, p. 2.

${ }^{19}$ Justin Godart, né à Lyon en 1871 et mort en 1956. Cet homme politique local et national, né un an avant Herriot et mort un an avant lui!, fut le rival malheureux d'Herriot à la mairie de Lyon. Si Augagneur ne met pas en' avant sa candidature en 1905, c'est qu'il voit en lui un rival potentiel, autrement plus dangereux qu'Herriot.

${ }^{20}$ Le Progrès, 12 avril 1904. 
ville de Lyon a supprimé totalement l'octroi. Faire disparaitre l'octroi, c'est réduire l'iniquité fiscale, puisque les mêmes droits étaient prélevés sur l'alimentation du pauvre et sur celle du riche, droits qui frappaient l'ouvrier dans la proportion de $5,40 \%$ de son salaire et seulement à la hauteur de $0,40 \%$ des revenus des gros propriétaires. Cette question de l'octroi donne lieu également à de nombreux rapports, dont celui d'avril $1904^{21}$, afin de faire taire les détracteurs qui ne peuvent être que des privilégiés. Organisée par la loi du 28 juin 1901, la suppression de l'octroi, véritable nouvelle nuit du 4 août à Lyon, n'a pas débouché sur un déficit des finances municipales comme le dénonçaient la presse et les parlementaires de Droite.

Les taxes de remplacement de l'octroi portent désormais sur les chevaux, les stalles des logeurs et marchands de chevaux, les automobiles, les débits de boissons, l'habitation, les propriétés bâties et non bâties, l'entretien des constructions, les locaux commerciaux, les cercles, sur les alcools, les spectacles et les constructions neuves. Elles ont donné aux finances municipales un bonus de 27000 francs en 1902 et de 188525,50 francs en 1903. La suppression de l'octroi a aussi amélioré la situation globale des contribuables lyonnais, puisque le montant des taxes de remplacement est moins élevé que le montant de l'octroi, soit une baisse d'impôt par contribuable de 3,72 francs. Cette baisse est même plus forte si l'on considère les contributions obligatoires ${ }^{22}$, puisqu'en 1899 , la contribution obligatoire par tête était de 20,10 francs et qu'elle n'est plus en 1904 que de 5,64 francs. Cette évolution, pour Augagneur, va dans le bon sens, puisqu'elle allège les contributions obligatoires payées par tous, donc par les plus pauvres - ceux-ci ne payent plus que la taxe d'habitation et encore les locataires des loyers de moins de 200 francs $^{23}$ ne sont soumis à aucune taxe de remplacement -, et aboutit à mettre plus de justice dans la répartition des charges communales. En effet, en chiffrant à 350000 habitants, le nombre de Lyonnais bénéficiaires de la baisse des impôts, la suppression des octrois a bien amélioré le bien-être du plus grand nombre, ce qui est l'objectif final de l'augagneurisme. En revanche, certaines catégories de contribuables ont vu leurs contributions augmenter, mais cela ne concerne que les contributions facultatives qui touchent les propriétaires, les commerçants, les propriétaires de voitures ou de chevaux, c'est-à-dire les contribuables souvent les plus riches. De plus, Augagneur démontre que la suppression de l'octroi, en baissant les prix, a amélioré la consommation des ménages, aussi bien en vin, dont la consommation est passée de 712000 hectolitres en 1900 à 932000 hectolitres en 1902, qu'en viande dont la consommation a augmenté de 5,613 kilogrammes par tête entre les mêmes dates.

\footnotetext{
${ }^{21}$ Victor AUGAGNEUR, Rapport sur les résultats de la suppression de l'octroi à Lyon présenté au Conseil municipal, Lyon 1904.

${ }^{22} \mathrm{Ce}$ sont les impôts qui frappent tous les contribuables, les contributions facultatives ne frappant que certaines catégories de contribuables.

${ }^{23}$ Soit 42300 loyers sur 135357 que compte la ville de Lyon.
} 
La deuxième grande réforme est la municipalisation des services municipaux. Pour reprendre une formule d'Augagneur, c'est cette réforme que "nos adversaires, avec des trémolos dans la voix, appellent le socialisme municipal et traitent d'expérience dangereuse"24. Augagneur dans sa conférence de 1903 différencie quatre classes dans les services publics dont une ville peut prendre la régie:

La première classe est celle des services de perception simple, ceux dans lesquels la commune touche une certaine somme de la part du contribuable, sans aucune compensation pour celui-ci. Pour Augagneur, une commune est tout à fait capable de percevoir directement ces taxes, ce que lui refuse les adversaires de la régie municipale, sans que le coût soit excessif et la fonctionnarisation trop forte. Il prend pour exemple la taxe de 0,10 franc prélevée à l'entrée sur chaque spectacle. Cette taxe a produit 180000 francs, soit plus d'un million de perceptions. Or, le coût de prélèvement ne dépasse pas $1,80 \%$ du produit, ce qui représente le traitement d'un employé municipal! Il conclut donc que la régie municipale revient moins cher aux contribuables que la perception en ferme et que payer correctement les employés municipaux n'a rien de scandaleux, même si pour "certains capitalistes trop intéressés la réduction du salaire est le premier des bénéfices de l'employeur"

La deuxième classe est celle des services de dépenses faites par la commune sans compensation par une recette quelconque. Le meilleur exemple est l'assistance publique qui, selon Augagneur, doit donner le plus d'assistance possible pour un minimum de dépenses possibles et ce pari peut très bien être tenu par la municipalité! Ce débat sur la municipalisation de l'assistance publique a lieu sur fond de suppression des congrégations. Le reproche que ses adversaires font à Augagneur, outre son anticléricalisme militant, porte sur le fait que la charité privée étant très économique, la prise en charge par les municipalités de ce genre de services va grever le budget municipal et, pour eux, l'Église demeure la seule institution capable d'assurer ce rôle. Pour apporter la contradiction, Augagneur prend l'exemple de l'orphelinat municipal de jeunes-filles de la CroixRousse, un des plus beaux de la région selon lui et, qui plus est, un orphelinat d'assistance et non de spéculation ${ }^{26}$. Le prix de journée pour l'année 1902 a été de 1,46 franc par tête et pour ce prix, les 51 jeunes-filles sont bien nourries, bien vêtues, bien soignées. Augagneur compare alors ce prix de journée ou celui de l'hospice Magnin-Fournet de la Croix-Rousse qui est de 1,67 franc à ceux du secteur privé religieux qui sont tous au-dessus et de conclure que "nous pouvons faire l'assistance directe dans de bonnes conditions" ${ }^{27}$, il confirme cet engagement public en parlant de l'asile de nuit de Perrache qui, pendant l'année 1902, a

\footnotetext{
${ }^{24}$ Le Progrès, le 12 avril 1904.

${ }^{25}$ Victor AUGAGNEUR, La régie directe des services publics par les municipalités, dans: PIC, GODART (dir.), Le mouvement économique 1905, p.75.

${ }^{26}$ Ibid., p. 80.

${ }^{27}$ Ibid.
} 
enregistré 23261 nuits à 0,37 franc l'unité. Augagneur a beau jeu de déclarer qu'il n'y a pas beaucoup de logeurs qui donneraient une soupe, un bain et un lit confortable propre pour ce prix ${ }^{28}$. Il termine sa démonstration en évoquant la régie municipale mise en place en 1903 pour l'enlèvement des boues des égouts et des immondices du parc. L'adjudicataire a été remplacé par un service des transports avec achat de chevaux, harnais, tombereaux et salaires des cochers, le tout se soldant par un bénéfice de 6000 francs pour la commune.

La troisième classe est celle des services de perception dans lesquels la recette prélevée sur le contribuable est compensée par un service rendu, c'est-à-dire lorsque l'opération a quelque chose de commercial, comme les droits de place sur la voie publique. Pour Augagneur, les adversaires de la régie municipale dénoncent l'arbitraire de l'agent car la somme à payer par le contribuable n'est pas déterminée à l'avance et, qui plus est, l'agent doit être honnête et faire preuve d'initiative. Si Augagneur souligne que des risques existent quant à l'agent, la régie municipale reste supérieure à la ferme. Il prend pour exemple les vogues ou fêtes populaires à Lyon. Autrefois, les droits payés par les forains étaient prélevés par un adjudicataire qui versait à la ville 42000 francs en 1890 , puis seulement 26000 en 1895. La ville touche en 1902, depuis qu'elle gère ce service en régie directe, 62000 francs et ses frais de perception ne sont que de 2500 francs, soit un bénéfice de 33500 francs par rapport à 1895 . Il en va de même des droits de place sur les marchés et des droits d'attache des bêtes de somme qui en 1900 rapportaient en fermage 396000 francs et qui en régie directe rapportent en 1902, pourtant une mauvaise année, 462000 francs moins 29080 francs de frais de perception, le bénéfice de la ville étant de 36320 francs. De plus, souligne Augagneur les traitements du personnel ont été augmentés. Lorqu'ils travaillaient pour l'adjudicataire, les agents touchaient 700 francs par mois, maintenant ils touchent 1000 francs pour un service qui va de 3 h à 9 heures du matin.

La quatrième et dernière classe est celle des services généraux communs, à forme commerciale ou industrielle, service des eaux, de l'éclairage, des abattoirs. Dans ce cas, il y a perception de la part de la commune et service rendu compensateur au contribuable. Cette quatrième classe dans la municipalisation des services publics est de loin celle qui pose les problèmes de gestion les plus délicats, celle qui prive le secteur privé des ressources les plus importantes et qui, de ce fait, mérite le plus la qualification de socialisme municipal. Augagneur en est conscient, puisqu'il l'évoque lui-même ${ }^{29}$. Une fois encore sa démonstration a pour but de prouver qu'une ville est capable de gérer elle-même ce genre de services sans ruiner les contribuables, voire même en allégeant la charge fiscale tout en dégageant pour la ville des recettes supplémentaires qu'elle peut consacrer à l'amélioration du sort des plus démunis. Municipaliser les services commerciaux et industriels, c'est priver les capitalistes des gras pâturages où les capitaux

${ }^{28}$ Ibid., p. 81 .

${ }^{29}$ Ibid., p. 73. 
paissaient librement et s'arrondissaient" ${ }^{\text {"30 }}$, puisque la situation de monopole était contraire à une baisse des prix. Il reprend sa méthode qui est d'asséner des exemples, chiffres à l'appui. Le cas des abattoirs est révélateur. Au temps de l'adjudication, la ville recevait 143000 francs et la Compagnie générale des abattoirs encaissait 182212 francs de bénéfices. La régie directe permet à la ville d'encaisser un bénéfice de 504733 francs pour 1902, soit 361733 francs de mieux. De plus, ajoute-t-il, dans les mêmes conditions que la compagnie, les taxes d'abattage ayant diminué depuis l'époque de l'adjudication et les quantités abattues ayant augmenté ce qui engendre des frais plus importants, les bénéfices de la municipalité auraient été de 724633 francs au lieu des 504733 francs. Cette comparaison prouve que la municipalité sait mieux gérer que la compagnie privée, mais aussi que de l'argent ne disparaît pas dans les poches de quelques administrateurs et qu'au lieu de profiter à quelques uns, il profite au plus grand nombre. En outre, les frais sont un peu inférieurs à ceux de la compagnie, ce qui infirme les accusations de fonctionnarisme de la municipalisation des services publics. Après les abattoirs, il prend l'exemple des théâtres qui est un service, par sa nature même, non rentable, d'où le peu d'intérêt d'une mairie pour une telle régie. Or, la régie municipale pour le théâtre des Célestins a dégagé un mince bénéfice, au lieu du déficit précédent et pour l'Opéra, le déficit s'est creusé compte tenu du fait qu'il a fallu investir en costumes et matériels, ce que l'ancien directeur n'avait pas fait. Pour justifier ce dérapage, Augagneur précise qu'un thêatre d'opéra ne peut jamais rapporter quelque chose au-delà de quelques milliers de francs ${ }^{31}$. Il termine sa démonstration en s'intéressant au service des eaux géré jusqu'à hier par la Compagnie générale des eaux, dont le siège est à Paris, et qui avait une concession de 90 ans, contrat que la municipalité Augagneur a racheté. Avant le bénéfice pour la commune était de 47356 francs, en 1902 la régie directe rapporte, avec le paiement de l'annuité de rachat à la Compagnie, 99804 francs, soit 52448 francs de plus. Ce bon résultat s'accompagne d'une multiplication des abonnés, donc de Lyonnais, pouvant bénéficier de l'eau.

Pour conclure sur l'œuvre réalisée, laissons la parole à Augagneur quand en 1904, à la brasserie Fritz, il évoque son bilan en ces termes: "On vous a dit que nous étions des socialistes? En avez-vous souffert vraiment? Vos intérêts ont-ils été lésés? Je vais plus loin, et je prétends que l'auraient-ils été, vous devriez l'accepter pour la grandeur du but et les résultats à en espérer"32.

\footnotetext{
${ }^{30}$ Ibid., p. 82.

${ }^{31}$ Ibid., p. 86.

${ }^{32}$ Le Progrès, 12 avril 1904.
} 


\section{Quelles lectures peut-on faire de l'augagneurisme?}

L'augagneurisme peut se résumer assez bien par cette formule choc: "La Compagnie cherchait à faire de grosses affaires, la Ville cherche à faire beaucoup d'affaires" ${ }^{33}$. Au-delà de cette formule, il y a un changement radical de la représentation du rôle de la municipalité dans la gestion des services publics. Avec le système de l'adjudication, la ville n'avait pas de compte à rendre au citadin qui n'était qu'un contribuable; avec l'avènement du socialisme municipal, les élus municipaux doivent rendre des comptes au citadin qui est désormais doté d'un rôle plus important en tant que contribuable-citoyen et surtout électeur. La gestion de la ville ne se fait plus en faveur des intérêts d'une minorité de capitalistes, représentés par des élites locales ou nationales, mais pour l'amélioration du bienêtre du plus grand nombre. On peut comprendre les violentes critiques émanant des anciens bénéficiaires qui se trouvent amputés d'une source de revenus non négligeable. En revanche, le socialisme municipal a la volonté de développer un personnel municipal fidélisé au pouvoir en place par des salaires plus élevés que dans le privé et par de meilleures conditions de travail, phénomène qui est dénoncé par les adversaires de cette politique comme étant une fonctionnarisation des municipalités, phénomène, bien évidemment, contraire, au discours libéral de la Droite.

L'augagneurisme, qui reprend les grandes lignes du socialisme municipal appliqué en Angleterre qui consiste à dégager des bénéfices afin de mener des actions dans le domaine social, est donc une méthode efficace et novatrice de gérer les villes, c'est-à-dire qu'avec le socialisme municipal les élus ne sont plus seulement des comptables des finances municipales qui reçoivent des recettes et mandatent des dépenses, c'est-à-dire des élus mineurs politiquement parlant, mais ils sont devenus de véritables responsables du devenir d'une ville et de ses habitants, avec l'objectif d'améliorer le bien-être du plus grand nombre et ne pas se contenter de reproduire les inégalités sociales existantes. Pour ce faire, ils mettent en place une politique volontariste qui commence, en tout premier lieu, par la maîtrise totale des ressources, seul moyen pour atteindre l'indépendance dans la prise de décision, qui est obtenue grâce aux régies municipales.

L'augagneurisme est-il pour autant synonyme de collectivisme, accusation lancée par ses adversaires pour le dénigrer et susciter chez les électeurs une attitude de rejet? Ce terme n'est pas le plus apte à définir le socialisme municipal d'Augagneur, même si celui-ci ne le renie pas; "Je ne me lave pas de l'épithète de collectiviste" ${ }^{94}$. La meilleure définition de l'augagneurisme est la volonté de lutter contre l'aristocratie de l'argent, bien sûr parce qu'elle est créatrice

\footnotetext{
${ }^{33}$ Augagneur, La régie directe 1905, p. 90.

${ }^{34}$ Ibid., p. 83.
} 
d'inégalités, mais aussi parce qu'elle est rebelle au progrès, seul capable d'apporter ce bien-être au plus grand nombre, idéal qui est au cœur du combat socialiste.

L'augagneurisme est ainsi un enjeu de pouvoir. Pour Augagneur, le socialisme municipal, c'est une manière républicaine - Augagneur parle de République sociale dans son discours d'investiture en $1904^{35}$, mais ce terme est-il synonyme dans sa bouche de socialiste? - et démocratique de gérer les villes face à la manière conservatrice et inégalitaire des adjudications mise en place par la Droite, mais aussi par le radical-opportuniste Gailleton. Avec son socialisme municipal, il règle ainsi ses comptes avec son vieil adversaire qui est toujours debout et candidat en 1904 et qui s'est opposé à ses méthodes modernes de gestion municipale qui ont même été à l'origine de son échec en $1896^{36}$. Il trouve curieux qu'en France, terre républicaine, cela soit considéré comme révolutionnaire, alors qu'en Angleterre ou en Allemagne, pays monarchiques, cette manière de gérer les finances municipales est acceptée depuis longtemps.

Enfin, l'augagneurisme consiste à renforcer le pouvoir du maire qui, tel un bourgmestre à l'allemande, est un gestionnaire avisé, pragmatique et doté d'une forte autorité, bref un véritable "proconsul". Si le socialisme municipal mis en place par Augagneur permet à Lyon de se démarquer de Paris qui vient d'élire une municipalité de Droite ${ }^{37}$, il n'est en aucune façon l'occasion d'affirmer un républicanisme municipal lyonnais face au pouvoir central. Augagneur ne recherche pas l'affrontement avec le préfet et pense que la mise en place des réformes se porte d'autant mieux, quand existe une collaboration fructueuse avec celui-ci. Or, depuis l'avènement de la III ${ }^{e}$ République, Lyon a transféré ses problèmes relationnels avec la République, problèmes hérités de la Révolution, vers l'antiparisianisme ${ }^{38}$. De ce fait, Augagneur ne partage pas ou ne veut pas partager la mémoire politique collective construite par les élites locales depuis plusieurs générations. Il n'incarne donc pas l'identité politique lyonnaise, puisqu'il se proclame socialiste et cherche à intégrer les communes périphériques peuplées de Rouges, alors que la ville est profondément modéro-centriste et se méfie, depuis 1793 , des Rouges. Il n'est pas non plus fédéraliste, cherchant à affirmer la république lyonnaise, et encore moins anti-parisianiste, puisque, par opportunisme, il regarde en 1905 vers Paris, ville capable de lui donner ce que Lyon est en train de lui reprendre, le pouvoir!

L'augagneurisme correspond bien à la fin de "l'Ancien Régime"39 pour la gestion municipale de Lyon. Cependant, l'augagneurisme, version lyonnaise du socialisme municipal, est aussi plus qu'une expérience, puisqu'il se veut le point

\footnotetext{
${ }^{35}$ A. M. L., Bulletin municipal officiel de la ville de Lyon, séance du 15 mai 1904.

${ }^{36}$ Augagneur déclare, ibid., p. 91 : "Je ne me consolerai jamais de n'avoir pas été élu en 1896".

${ }^{37}$ Le Façonné de Guignol écrit le 18 mai 1900: "Pendant que Paris s'agenouille devant le SacréCœur et se coiffe d'un éteignoir, notre grande ville prend le bonnet rouge de la liberté"!

${ }^{38}$ Voir supra note 2.

${ }^{39}$ Ibid., p. 71 .
} 
de départ d'une nouvelle expression de la gouvernance municipale. Herriot l'a bien compris et l'œuvre municipale de ce maire est dans la continuité de la politique initiée par Augagneur en gommant l'appellation "socialiste". Le bilan ${ }^{40}$ qu'il présente en 1925, après plus de vingt ans de pouvoir local, aurait pu être approuvé par Augagneur, les élus sont de même origine politique, les socialistes en moins.

L'augagneurisme peut donc être lu comme une expérience par sa brieveté et par son affichage socialiste. Cette étiquette collée sur l'hôtel-de-ville ne s'est vue que rarement à Lyon, aussi bien avant, excepté lors de violences collectives à caractère révolutionnaire, qu'après, du moins au cours du $\mathrm{XX}^{\mathrm{e}}$ siècle!

\section{Deutsche Zusammenfassung}

Nach den Kommunalwahlen von 1900 wurde Victor Augagneur, Chirurg und Professor an der medizinischen Fakultät, zum Bürgermeister von Lyon gewählt. Er profitierte dabei vom politischen Klimawechsel nach der Dreyfus-Affäre, als die wieder auftretenden klerikalen/antiklerikalen Spannungen den Konsens von politischer Mitte und gemäßigten Republikanern sprengten, von dem der seit den Kommunal-Gesetzen von 1881/84 amtierende radikal-opportunistische Bürgermeister Antoine Gailleton profitiert hatte. 1904, im Jahr seiner Wiederwahl in Lyon, wird Augagneur auch zum Abgeordneten gewăhlt und sitzt in der Nationalversammlung an der Seite von Jaurès und Guesde. Allerdings schließt er sich im April 1905 nicht der SFIO an, um sich nicht von den Republikanern in Lyon zu trennen, die jeder Verankerung auf der Extremen, die sie an das Drama von 1793 erinnert, mißtrauen. Aus diesem Grund verliert Augagneur die Unterstützung der Sozialisten, ohne indes die der gemäßigten Republikaner zu gewinnen. Zudem hat ihn sein autoritäres Verhalten, man spricht von einem "Prokonsulat", wenig beliebt gemacht. Angesichts der sich zeigenden Ausweglosigkeit behält sein Opportunismus die Oberhand über seine politische Militanz. Er tritt 1905 zurück, um Gouverneur von Madagaskar zu werden, hat aber Hoffnung, eines Tages wieder das Bürgermeisteramt zurückzugewinnen. Das Problem dabei ist allerdings sein Nachfolger, den er selbst designiert hat und der kein geringerer als Édouard Herriot ist. Augagneurs Programm umfasst die Abschaffung des Oktroi, der auf ungerechte Weise die "classe des ouvriers de l'industrie" treffe, und dessen Ersetzung durch Steuem für die "classe des propriétaires", die Schaffung von Gemeindebetrieben, solide öffentliche Finanzen und den Ausbau des weltlichen Schulwesens. Seine Gegner geben diesem Programm das Etikett Munizipal-Sozialismus. Victor Augagneur hingegen meint, seine Stadt ebenso korrekt zu verwalten, wie es Kapitaleigner mit ihrer Aktiengesellschaft tun. Er ist mitnichten sozialistischer Theoretiker oder gar Sozialist. Aus diesem Gegensatz zwischen der Person und ihrem Handeln rührt die Kurzlebigkeit des Augagneurismus her und sein Charakter eines Experiments. Dennoch wird sein Programm unter anderem Namen wieder aufgenommen: von seinem Nachfolger Herriot, der 52 Jahre lang Bürgermeister von Lyon bleiben wird.

\section{English Abstract}

Victor Augagneur, surgeon and professor of medicine at the university, is elected mayor of Lyon after the municipal elections of 1900 . To be elected, he has benefited by the Dreyfus' case. This

${ }^{40}$ Édouard HERRIOT, L'œuvre municipale 1905-1929, Lyon 1929. 
case has roused the clerical/anticlerical tensions and has broken the centrist and republican consensus which benefits to the radical-opportunist mayor, Antoine Gailleton who was in place since the municipal laws of 1881-1884. In 1904, year of his reelection at the Lyons' town council, he is voted in as a deputy and sits at the french national Assembly, beside Guesde and Jaurès. Nevertheless, he doesn't join the SFIO in april 1905 in order not to be cut off from the Lyons' republicans who are anxious about any extreme position which remembers them 1793' tragedy. Therefore, Augagneur loses the socialists' support, without having the moderate republicans' one. Furthermore, his authoritarianism get talked about "proconsulship" has made him impopular. In face of such problems, his opportunism prevails over his militancy. He resigns in 1905 to become governor of Madagascar, hoping to recover the towns mayoralty. The problem is that the successor he has named is nobody else than Edouard Herriot. Augagneurs program turns on the removal of the granting which hits "the class of industry's workers" in an unfair way and on its replacement by taxes "on the class of owners", on the creation of municipal government control, on save public finances and on the growth of state education. The mayor's opposents give this program the name of municipal socialism. According to Augagneur, he manages the city as properly as shareholders do with limited companies. Victor Augagneur has nothing of a socialist theoretician or even of a socialist. From this antagonism between the man and his acts follows the briefness of the augagneurism and a guarantee of experience. Nevertheless, Augagneur's program will be used again, under an another name, by Herriot his successor who will stay mayor during 52 years. 